\title{
Die Langzeitperspektive in der Richtlinienpsychotherapie: Rehabilitationspsychotherapie
}

\author{
Michael Linden \\ Forschungsgruppe Psychosomatische Medizin an der Charité Universitätsmedizin Berlin und Institut für Verhaltenstherapie Berlin, \\ Berlin, Deutschland
}

In dieser Ausgabe der Verhaltenstherapie findet sich ein Beitrag von Voderholzer und Barton [2016] zum Thema «Anhaltende Wirkung von Psychotherapie bei Depression». Die Psychotherapie wird häufig als Maßnahme zur Behandlung akuter Erkrankungszustände diskutiert; dazu liegen auch die meisten empirischen Wirksamkeitsstudien vor. Dies ist in der Psychotherapie nicht anders als in anderen Therapiebereichen, wie z.B. der Pharmakotherapie.

Dieser Fokus auf der Behandlung von «Krankheitsepisoden» hat in der angewandten Therapie aber nur eine geringe Bedeutung. So klingen akute depressive Episoden in vielen Fällen nach einigen Wochen spontan $\mathrm{ab}$, sodass psychotherapeutische Behandlungen, die sich in der Regel über viele Monate erstrecken, erst zur Wirkung kommen können, wenn die Spontanremission längst erfolgt ist. Sieht man einmal von psychotherapeutischer Krisenintervention ab, dann ergibt sich daraus, dass die Psychotherapie im engeren Sinne vorrangig bei Krankheiten indiziert ist, die länger als ein halbes Jahr anhalten - nach der Definition von $₫ 2$ des Sozialgesetzbuchs IX sind damit die Kriterien einer Behinderung erfüllt.

Diese Langzeitorientierung gilt erst recht für die sogenannte «Richtlinienpsychotherapie», die in Deutschland die Regel in der ambulanten Psychotherapieanwendung ist. Sie ist aufgrund der vorgegebenen organisatorischen Rahmenbedingungen ausschließlich bei chronischen Erkrankungen durchführbar. Die Patienten müssen sich bei einem Therapeuten anmelden und im Normalfall Wochen, wenn nicht Monate, auf einen Therapieplatz warten. Anschließend müssen mehrere probatorische Sitzungen erfolgen, was 1-2 Monate in Anspruch nimmt. Dann müssen ein therapeutischer Bericht verfasst und ein Antrag gestellt werden, der von den Kostenträgern an einen Gutachter weitergeleitet wird, auf dessen Empfehlung hin eine Kostenübernahme bewilligt wird. Dieser Prozess dauert wiederum Wochen. Danach wird nur ein befristetes Stundenkontingent befürwortet, was bedeutet, dass die Behandlung, selbst wenn eine zusätzliche und vorübergehende Verlängerung erfolgt, zum Ende der bewilligten Stunden zu beenden ist, unabhängig davon, wie es dem Patienten geht. Dieses Vorgehen in der ambulanten Richtlinienpsychotherapie gleicht dem bei stationären Rehabilitationsmaßnahmen. Hinzu kommt, dass der Kontakt zum Therapeuten während der Therapiedurchführung weitgehend auf die verabredeten Sitzungen beschränkt ist und ansonsten bestenfalls Telefongespräche zu sehr eingeschränkten Zeiten stattfinden können. All dies ist therapeutisch, juristisch und ethisch nur bei chronischen Erkrankungen erlaubt. Bei einer akuten depressiven Episode, gar noch mit akuter Suizidalität, wäre es ein Kunstfehler, ein solches Behandlungsregime anzuwenden. Bei akuten Erkrankungen gilt zudem, dass eine Behandlung so lange zu dauern hat, wie eine therapeutische Notwendigkeit besteht, während es bei chronischen Krankheitszuständen keine «unterlassene Hilfeleistung» gibt. Nur deshalb ist es beispielsweise möglich, dass ein Patient monatelang auf den Beginn der Therapie wartet oder der Kontakt zum Therapeuten beendet wird, obwohl der Patient weiterhin schwer krank ist.

Allerdings gibt es trotz der genannten Einschränkungen für Richtlinienpsychotherapeuten genug zu tun. Hirnorganische Störungen, Suchterkrankungen, schizophrene Erkrankungen, depressive Störungen, Angststörungen, somatoforme Störungen oder Persönlichkeitsstörungen sind zu einem großen Teil, und manchmal sogar per definitionem, chronische Krankheiten bzw. «psychische Behinderungen». Es geht also nicht um die Behandlung von «Krankheitsepisoden», sondern von «Krankheitsentwicklungen». Dies ist sogar für einige Therapien namensgebend, wie z.B. die Dialektisch-Behaviorale Therapie (DBT), die deshalb «dialektisch» heißt, weil das Grundaxiom darin besteht, dass Borderlinestörungen nicht zu heilen, aber dennoch zu behandeln sind.

Akzeptiert man die Realität, dass psychische Krankheiten ihrer Natur nach, unter therapeutischen Überlegungen und auch nach geltendem Sozialrecht gemäß $\$ 2$ und $\$ 26$ SGB IX in einer Vielzahl von Fällen als chronische Krankheiten und damit als psychische Behinderungen anzusehen sind, dann ergibt sich daraus eine Reihe unmittelbarer Konsequenzen:

\section{KARGER}

Fax +497614520714
(๑) 2016 S. Karger GmbH, Freiburg

$1016-6262 / 16 / 0262-0080 \$ 39.50 / 0$
Prof. Dr. Michael Linden

Forschungsgruppe Psychosomatische Medizin an der Charité Universitätsmedizin Berlin und Institut für Verhaltenstherapie Berlin

Hindenburgdamm 30, 12200 Berlin, Deutschland

michael.linden@charite.de 
- Die Indikation für eine Richtlinienpsychotherapie ist nicht von der aktuellen Krankheitsschwere abhängig, sondern von der Prognose hinsichtlich überdauernder Wirkungen nach Abschluss von einem halben bis 2 Jahren Psychotherapie;

- die Therapiewirkung ist nicht nur an der Besserung des psychischen Befunds (bzw. der Funktionsstörung nach der International Classification of Functioning, Disability and Health (ICF)) zu bemessen, sondern muß «ganzheitlich» ansetzen, d.h. nach den Vorgaben der ICF auch «Capacities», «Kontext» und «Teilhabe» im Auge haben;

- da die behandelten Krankheiten, wie z.B. eine Borderlinestörung, eine generalisierte Angststörung oder Suchterkrankung, auch nach 2 Jahren Psychotherapie nicht verschwinden, muss sich die Richtlinienpsychotherapie in die Langzeitbetreuung der Patienten im Rahmen der sogenannten Grundversorgung einordnen; die Richtlinienpsychotherapie ist durchzuführen als intervalläre Behandlung im Kontext einer Langzeitbetreuung und muss daher dem «Primärbehandler» zuarbeiten bzw. sich mit diesem «koordinieren».

Die Arbeit von Voderholzer und Barton [2016] zeigt am Beispiel depressiver Erkrankungen, dass die Psychotherapie durchaus dem Anspruch gerecht werden kann, überdauernde Wirkungen zu haben. Allerdings zeigt die Übersicht über die vorliegende Literatur auch, dass die meisten Studien nur auf die Symptomatik Bezug nehmen. Fähigkeiten oder Teilhabe kommen nicht vor. Das ist eine Perspektivverkürzung, die nicht nur den Patienten und ihren Erkrankungen nicht gerecht wird, sondern auch der Psychotherapie selbst. Die Psychotherapie ist geradezu die Methode der Wahl, um bei chronischen Patienten die Krankheitsbewältigung zu unterstützen, also z.B. Skills zu vermitteln zur Bewältigung der unbeeinflussbaren Affektlabilität bei Borderlinestörungen, oder Fähigkeiten zu trainieren zur Förderung der sozialen Teilhabe von Patienten, die unter nicht beseitigbaren schizophrenen Erkrankungen leiden, oder zur Herstellung von Kontext- bzw. Umweltbedingungen, um bei Kindern eine schulische Inklusion trotz nicht zu bessernder ADHS zu ermöglichen.

Voderholzer und Barton [2016] haben also Recht, wenn sie schreiben, dass «zusätzlich zu Aussagen zur Symptomatik auch Daten zum allgemeinen Funktionsniveau und zur Lebensqualität von Interesse» wären. Die Psychotherapie hat nicht nur Wirkungen im Sinne einer «Efficacy», sondern auch einer «Efficiency». Das sollte in der Behandlung des einzelnen Patienten, bei den vorgegebenen Therapiezielen und auch der Forschungsmethodik Berücksichtigung finden. Ein Verständnis von Psychotherapie allgemein und Richtlinienpsychotherapie im Besonderen als «Rehabilitationspsychotherapie» eröffnet wichtige ergänzende Perspektiven sowohl im Individualfall als auch bei der Entwicklung von Psychotherapiekonzepten insgesamt.

\section{Literatur}

Voderholzer U, Barton B: Langfristige Wirkung von Psychotherapie bei nichtchronischen Depressionen: Ein systematisches Review von Studien im Vergleich mit Pharmakotherapie. Verhaltenstherapie 2016;26:108115 . 